\title{
CONCOMITÂNCIA DA TIREOIDITE DE HASHIMOTO E O CARCINOMA DIFERENCIADODA TIREÓIDE
}

\author{
CONCOMITANCE OF HASHIMOTO'S THYROIDITIS AND DIFFERENTIATED THYROID \\ CANCER
}

\author{
Alexandre Roberti ${ }^{1}$; Josias de Andrade Sobrinho - ECBC ${ }^{2}$; \\ Odilon Victor Porto Denardin²; Abrão Rapoport- ECBC ${ }^{3}$
}

\begin{abstract}
RESUMO: Objetivo: Avaliar a associação da tireoidite auto-imune e o carcinoma diferenciado da tireóide na Santa Casa de Misericórdia de Goiânia-Goiás. Método: De 1395 tireoidectomias realizadas de 1994 a 2003, foram selecionadas 120 carcinomas diferenciados ( 27 foliculares e 93 papilíferos). Foram avaliadas as variáveis clínicas (idade e sexo) com apresentação de freqüências e de sumários de medidas-resumo na descrição estatística. Para aferir a associação de tireoidite auto-imune e carcinoma diferenciado da tireóide em função dos achados histopatológicos, utilizou-se tabelas de contingência e análise pelo teste não paramétrico do quiquadrado de Pearson. Em todos os testes estatísticos foi adotado um nível de significância de 5\% (p<0,05). Resultados: $11,1 \%$ dos carcinomas foliculares e $18,3 \%$ dos carcinomas papilíferos estão associados à tireoidite auto-imune. Existe uma relação de freqüências quatro vezes maior da tireoidite auto-imune com o carcinoma diferenciado da tireóide em comparação com outras doenças tireoideanas $(16,7 \%$ X 3,6\%). Conclusão: Tais resultados permitem inferir que a associação entre a tireoidite auto-imune e o carcinoma diferenciado da tireóide é mais que casual, exigindo uma observação clínico-laboratorial cuidadosa nos portadores da doença auto-imune (Rev. Col. Bras. Cir. 2006; 33(6): 345-349).
\end{abstract}

Descritores: Tireoidite; Neoplasias da glândula tireóide; Tireoidite auto-imune.

\section{INTRODUÇÃO}

A incidência do câncer da tireóide mostra grandes diferenças entre os países e populações em regiões endêmicas sendo sua incidência e mortalidade pelo câncer da tireóide, consideradas maiores que em regiões não endêmicas ${ }^{1}$.

Os infiltrados linfocitários da tireóide são encontrados em uma grande variedade de doenças: tireoidite de Hashimoto, tireoidite linfocítica, tireotoxicose, bócio nodular e no câncer da tireóide. Os graus de substituição do parênquima tireoidiano por linfócitos variam de infiltrados difusos a pequenas alterações focais, encontradas nos bócio nodulares e carcinomas ${ }^{2}$, sendo que o nome tireoidite de Hashimoto tem persistido na literatura para descrever a "tireoidite auto-imune", termo usado indevidamente nos casos com tireoidite focal ou generalizada, e deve ser empregado exclusivamente para descrever pacientes com bócio, hipotireoidismo que demonstrem altos títulos de anticorpos antitireóide ou presença de citologia e/ou biópsia comprovando a enfermidade.

A incidência de tireoidite de Hashimoto em associação com o carcinoma da tireóide permanece controversa, não se encontrando evidências de que o carcinoma tireoidiano seja originado no epitélio proliferado da tireoidite de Hashimoto. As neoplasias com baixo grau de malignida- de (carcinoma papilífero e folicular) ocorriam com maior freqüência nos casos de tireoidite de Hashimoto do que nos carcinomas não papilares e anaplásicos, justificada pela incidência mais comum das neoplasias de baixo grau de malignidade comparativamente àquelas de alto grau, e por afetar um grupo mais jovem de pacientes como foi detectado com a tireoidite de Hashimoto. Há evidências que a reação inflamatória auto-imune e a presença de auto-anticorpos podem retardar o crescimento e a disseminação do carcinoma da glândula tireóide ${ }^{3,4}$. Não ocorre o aumento nas taxas de associação com malignidade quando comparamos tireoidite auto-imune com a população em geral, com infiltração linfocítica em cortes histológicos, especialmente em casos malignos, não significando de rotina a associação com a doença auto-imune ${ }^{5}$.

Existem evidências indiretas que suportam a idéia da associação da tireoidite de Hashimoto e do carcinoma da tireóide, como o aumento de 2,77 vezes da freqüência da tireoidite de Hashimoto associada ao carcinoma papilar quando comparada ao grupo controle ${ }^{6}$. Todavia, os rearranjos do oncogene RET/PTC eram eventos precoces no desenvolvimento do carcinoma papilar da tireóide, podendo estar presente nos tirócitos normais das regiões infiltratadas por linfócitos, podendo ser mutações advindas a fatores genéticos desconhecidos ou fatores ambientais ${ }^{7}$.

1. Mestre pelo Curso de Pós-Graduação em Ciências da Saúde do Hospital Heliópolis, Hosphel, São Paulo-SP.

2. Docente Permanente do Curso de Pós-Graduação em Ciências da Saúde do Hospital Heliópolis, Hosphel, São Paulo-SP.

3. Coordenador do Curso de Pós-Graduação em Ciências da Saúde do Hospital Heliópolis, Hosphel, São Paulo-SP.

Recebido em 29/05/06

Aceito para publicação em 30/06/06

Fonte de financiamento: nenhuma

Conflito de interesses: Não há.

Trabalho realizado no Serviço de Cabeça e Pescoço da Santa Casa de Misericórdia de Goiânia da Universidade Católica de Goiás. 
O conceito de inter-relacionamento entre doenças inflamatórias e neoplásicas, está bem estabelecido em certos tecidos mas controverso na tireóide, como o aumento do risco da ocorrência do carcinoma diferenciado em pacientes com tireoidite de Hashimoto. Houve uma freqüente detecção do p63 no carcinoma papilar e na tireoidite de Hashimoto, em contraste com uma expressão negativa em outras neoplasias e na doença de Graves, achados que levantaram a possibilidade destas duas doenças estarem associados ${ }^{8}$.

A justificativa deste trabalho foi a intensa relação entre os carcinomas: folicular e papilar e a tireoidite auto-imune, com as mudanças induzidas pelo homem no meio ambiente. Nas ultimas décadas, na Região Centro-Oeste do Brasil, houve um esforço governamental fazendo variar a ingestão de iodo da população, fato que justificou a avaliação da associação entre tireoidite auto-imune e o carcinoma diferenciado da tireóide em uma série de pacientes submetidos à tireoidectomia na Região Centro-Oeste do Brasil.

\section{MÉTODO}

Foram analisados os prontuários de 1395 pacientes submetidos à tireoidectomia entre janeiro de 1994 e dezembro de 2003, no Serviço de Cirurgia de Cabeça e Pescoço da Santa Casa de Misericórdia de Goiânia - Hospital Escola da Universidade Católica de Goiás.

Foram analisados os fatores epidemiológicos, a apresentação morfológica da doença, os diagnósticos e os resultados dos exames anátomo-patológicos. As indicações e a técnica operatória utilizada para a realização da tireoidectomia também foram estudados

Após a revisão os dados foram compilados em uma planilha do Microsoft Excel, onde os cálculos foram realizados. Inicialmente as variáveis clínicas (sexo e idade) foram analisadas descritivamente com a apresentação de freqüências e de sumários de medidas-resumo (amplitude, média e freqüência relativa), na descrição casuística.
Para avaliar a existência de associações entre os achados histopatológicos de tireoidite auto-imune e de carcinomas diferenciados da tireóide, utilizou-se a descrição em tabelas de contingência e análise pelo teste não paramétrico do qui-quadrado de Pearson.

Em todos os testes estatísticos foi adotado um nível de significância de $5 \%(\mathrm{p}<0,05)$.

\section{RESULTADOS}

Foram realizadas 1395 tireoidectomias sendo 1256 (90\%) em pacientes do sexo feminino e 139 (10\%) em pacientes do sexo masculino. As idades variaram de 5 a 92 anos com uma média de 50 anos. Quanto à apresentação morfológica da doença, obtivemos 85 (6\%) de bócios difusos, 809 (58\%) bócios multinodulares e 501 (36\%) bócios uninodulares.

Encontramos 1612 diagnósticos de doenças diferentes. Os resultados dos exames anátomo-patológicos foram: a) Adenoma folicular $155(9,6 \%)$; b) Outros adenomas 37 (2,3\%); c) Bócio adenomatoso 87 (5,4\%); d) Bócio colóide 982 (60,9\%); e) Carcinoma folicular 27 (1,7\%); f) Carcinoma papilar $93(5,8 \%)$; g) Cistos nove $(0,6 \%)$; h) Bócio difuso tóxico $69(4,3 \%)$; i) Outras tireoidites $6(0,4 \%) ; \mathrm{j})$ Outras neoplasias malignas 16 $(1,0 \%)$ e l) Tireoidite Auto-imune 131 (8,1\%).

Selecionamos os pacientes com diagnóstico de carcinoma folicular e carcinoma papilar. Com carcinoma folicular foram 27 pacientes, $23(85,2 \%)$ do sexo feminino e $4(14,8 \%)$ do sexo masculino, com idade variando de 16 a 88 anos, com uma média de 54,3 anos.

Quanto à apresentação morfológica obtivemos 15 $(55,5 \%)$ pacientes com bócio uninodular, 10 (37\%) pacientes com bócio multinodular e $2(7,5 \%)$ pacientes com bócio difuso. Com diagnóstico de carcinoma papilar foram 93 pacientes, 81 (87\%) do sexo feminino e 12 (13\%) do sexo masculino, com idade variando de 16 a 76 anos com uma média de 43,3 anos. Quanto à apresentação morfológica obtivemos 50 (53,7\%) pacientes com bócio uninodular, 39 (42\%) pacientes com bócio multinodular e 4 (4,3\%) pacientes com bócio difuso.

Tabela 1 - Freqüencia de tireoidite auto-imune no carcinoma folicular.

\begin{tabular}{lcccccc}
\hline & \multicolumn{2}{l}{ Tireoidite auto-imune } & & & & \\
& sim & $\mathbf{( \% )}$ & não & (\%) & Total & (\%) \\
\hline Carcinoma folicular & 3 & 11,1 & 24 & 88,9 & 27 & 100 \\
Outros diagnósticos & 54 & 3,6 & 1438 & 96,4 & 1492 & 100 \\
Total & 57 & 3,8 & 1462 & 96,2 & 1519 & 100 \\
\hline
\end{tabular}

$X^{2}=4,12$

$p=0,04$

Tabela 2 - Freqüência de tireoidite auto-imune no carcinoma papilar.

\begin{tabular}{llrcrrr}
\hline & \multicolumn{2}{l}{ Tireoidite auto-imune } & nã & (\%) & Total & (\%) \\
\hline Sim & $\mathbf{( \% )}$ & 18,3 & 76 & 81,7 & 93 & 100 \\
Outrinoma papilar & 17 & 3,6 & 1438 & 96,4 & 1492 & 100 \\
Total & 54 & 4,5 & 1514 & 95,5 & 1585 & 100 \\
\hline
\end{tabular}


Nos 27 pacientes com carcinoma folicular encontramos $24(88,9 \%)$ sem associação com a tireoidite auto-imune e três $(11,1 \%)$ com associação da tireoidite auto-imune (Tabela 1).

Nos 93 pacientes com carcinoma papilar encontramos $76(81,7 \%)$ sem associação com a tireoidite auto-imune e $17(18,3 \%)$ com associação da tireoidite auto-imune (Tabela 2).

Comparamos os resultados de freqüência de associação com tireoidite auto-imune nos carcinomas diferenciados entre si (Tabela 3).

Nos 1492 diagnósticos sem carcinomas diferenciados da tireóide encontramos 1438 (96,4\%) sem associação da tireoidite auto-imune e 54 (3,6\%) com associação da tireoidite auto-imune (Tabela 4).

As tireoidectomias foram indicadas utilizando os critérios clássicos: a) bócios tóxicos; b) bócios simples com suspeita de malignidade; c) bócio simples com sinais ou sintomas de compressão de estruturas do pescoço; d) bócios mergulhantes ou intratorácicos; e) por razões estéticas; f) neoplasias tireoidianas.

Em todos os pacientes a rotina cirúrgica foi a mesma: a) paciente em decúbito dorsal horizontal; b) anestesia geral; c) abertura da pele e tecido celular subcutâneo com a incisão clássica em colar, tipo Kocher; d) descolamento dos retalhos cutâneos superior e inferior ao nível do músculo platisma; e) abertura da rafe mediana longitudinalmente.

Nos bócios nodulares unilaterais todo o lobo correspondente foi retirado e nos bilaterais toda a tireóide foi extirpada. Excepcionalmente nos nódulos únicos de istmo, a istmectomia foi realizada. Nos bócios tóxicos o procedimento executado foi lobectomia total unilateral e parcial contra-lateral. Todos os pacientes foram drenados com dreno tubular. A síntese da ferida operatória foi realizada por planos.

\section{DISCUSSÃO}

A tireoidite auto-imune é geralmente considerada um processo inflamatório e degenerativo. Em algumas tireóides a tireoidite se desenvolve secundariamente ao crescimento e invasão tumoral. Esta reação inflamatória do parênquima tireoidiano e da neoplasia ocorre mais frequentemente no carcinoma papilar. Este fato sugere que a reação inflamatória é derivada de antígenos oriundos da própria neoplasia e não da invasão tecidual normal. Sugere ainda, que o tecido do carcinoma papilar da tireóide seja mais parecido antigenicamente ao tecido tireoidiano normal, quando comparado com o carcinoma folicular? .

Sendo processos de múltiplos passos, tireoidite autoimune e carcinoma diferenciado da tireóide sofrem influências de muitos fatores. Entre estes fatores, dois exercem papel fundamental em ambos os processos, a ingestão quantitativa de iodo e a estimulação pelo TSH. Após a introdução da terapêutica e profilaxia com iodo ocorre um aumento da incidência de tireoidites ${ }^{10}$. A distribuição dos diferentes tipos de carcinomas diferenciados da tireóide parece ser influenciada pela ingestão de iodo da população, com mais carcinomas foliculares nas áreas iodo-deficientes e mais carcinomas papilares em áreas mais ricas em iodo. Populações que iniciam a iodo profilaxia demonstram aumento dos carcinomas ${ }^{11}$. A incidência do carcinoma papilar está aumentando na maioria dos países nos últimos 60 anos, fato que deve estar relacionado com a ingestão de iodo ${ }^{12,13}$. No mundo existem locais com maior e menor incidência de câncer da tireóide, a diferença na ingestão de iodo pode ser um fator explicativo para estas diferenças geográficas. Ingestão aumentada de iodo está associada com um pequeno aumento da incidência de câncer da tireóide. Com a iodação há risco de aumentar a incidência de câncer, os programas de iodação devem ter supervisão rigoro$\mathrm{sa}^{14}$. Estudos têm demonstrado que em locais de iodo-deficiência com a profilaxia, tem se desenvolvido nestas populações um excesso de ingestão do iodo ${ }^{15}$.

Na nossa casuística, nos 27 pacientes com carcinoma folicular encontramos $24(88,9 \%)$ sem associação com a tireoidite auto-imune e $3(11,1 \%)$ com associação da tireoidite auto-imune (Tabela 1), enquanto que na literatura ${ }^{5,6,16}$ há referências desta associação que varia de $0,8 \%$ a $19 \%$.

Tabela 3 - Freqüência de tireoidite auto-imune nos carcinomas papilar e folicular.

\begin{tabular}{lcccccc}
\hline & \multicolumn{2}{l}{ Tireoidite auto-imune } & Nã & (\%) & Total & $\mathbf{( \% )}$ \\
\hline Sim & $\mathbf{( \% )}$ & 18,3 & 76 & 81,7 & 93 & 100 \\
Carcinoma papilar & 17 & 11,1 & 24 & 88,9 & 27 & 100 \\
Total & 3 & 16,7 & 100 & 83,3 & 120 & 100 \\
\hline \multicolumn{7}{c}{$p=0,374$} \\
\hline
\end{tabular}

Tabela 4 - Freqüência de tireoidite auto-imune nos carcinomas diferenciados.

\begin{tabular}{lcrrrrr}
\hline & \multicolumn{2}{c}{ Tireoidite auto-imune } & & & & \\
& sim & $\mathbf{( \% )}$ & Não & $\mathbf{( \% )}$ & Total & (\%) \\
\hline Carcinoma diferenciado & 20 & 16,7 & 100 & 83,3 & 120 & 100 \\
Outros diagnósticos & 54 & 3,6 & 1438 & 96,4 & 1492 & 100 \\
Total & 74 & 4,6 & 1538 & 95,4 & 1612 & 100 \\
\hline
\end{tabular}


Nos 93 pacientes com carcinoma papilar encontramos $76(81,7 \%)$ sem associação com a tireoidite auto-imune e 17 (18,3\%) com associação da tireoidite auto-imune (Tabela 2). A associação entre a tireoidite auto-imune e o carcinoma papilar da tireóide permanece controversa, sendo aceita por uns ${ }^{6,9}$ e negada por outros ${ }^{5}$. Em nossa casuística existiu uma associação entre a tireoidite auto-imune e o carcinoma papilar de 18,3\% (Tabela 2).

Quanto à associação entre o carcinoma papilífero e a tireoidite de Hashimoto, há descrições que revelam 30\% de infiltrações mínima de parênquima tireoideano e $9 \%$ de infiltrados linfocíticos difusos ${ }^{16}$, enquanto que são descritos associações que variam de $3,5 \%$ a $76 \%{ }^{6,9,16,17}$.

Nos 120 pacientes com carcinoma diferenciado da tireóide encontramos 100 (83,3\%) sem associação com a tireoidite auto-imune e 20 (16,7\%) com associação da tireoidite auto-imune (tabela 3). Verificamos que não há diferença estatisticamente significante $(\mathrm{p}=0,374)$ entre eles. Quanto à literatura, observamos que os autores ${ }^{4,18}$, descreveram associações entre os carcinomas diferenciados da tireóide e tireoidite autoimune, que variaram de $1,2 \%$ a $17,7 \%$ dos casos. Quanto à associação da tireoidite auto-imune com carcinomas diferenciados e outras patologias, em nossa casuística tivemos novamente uma significante associação em 16,7\% dos casos, sugerindo que a mesma deva ser mais que casual (Tabela 4). Outros autores ${ }^{19}$, estudaram pacientes com carcinoma diferenciado da tireóide e controles com bócio colóide pareando através da mesma idade e sexo, encontrando nos casos de carcinoma tireoidiano a associação com tireoidite auto-imune em $38 \%$ dos pacientes e nos controles, a associação de $11 \%$. A diferença foi considerada estatisticamente significante. As freqüências encontradas no presente estudo estão de acordo com o trabalho citado $^{19}$, quando encontramos nos casos de carcinoma diferenciado da tireóide uma associação com tireoidite auto-imune em $16,7 \%$ e nas outras patologias uma associação de apenas 3,6\%, com uma diferença significante $(\mathrm{p}<0,001)$ entre elas (Tabela 4).

A histologia convencional sempre foi considerada um pilar no diagnóstico dos carcinomas da tireóide. Recentemente, a imunohistoquímica vem demonstrando técnicas que podem auxiliar como adjuvantes no diagnóstico. O melhor método para o diagnóstico do carcinoma papilar está relacionado com o rearranjamento RET/PTC. Rearranjamentos do RET levam a uma expressão gênica, isto ocorre em aproximadamente $50 \%$ dos carcinomas papilares ${ }^{20}$. Porém, a tireoidite auto-imune e o carcinoma papilar da tireóide se misturam quanto à morfologia, modelo imunohistoquímico e perfil molecular. Mesmo sendo considerada uma condição benigna a tireoidite auto-imune pode evidenciar rearranjamento do RET/PTC ${ }^{20}$

Outra característica interessante é a detecção freqüente do p63 em tireoidite auto-imune e carcinoma tireoidiano em contraste com a sua detecção incomum nos pacientes com outras neoplasias e na doença de Graves. Estes achados indicam a possibilidade destas doenças se inter-relacionarem ${ }^{8}$.

A associação de carcinoma papilar e tireoidite autoimune deve ser mais que casual nos indivíduos susceptíveis, considerada a história familiar da tireoidite de Hashimoto ${ }^{21}$. Podemos conceber que os mesmos estímulos que contribuem à tireoidite auto-imune podem agir como iniciadores do carcinoma diferenciado da tireóide ${ }^{18}$. Nossos achados nos permitem sugerir que o controle clínico e laboratorial dos pacientes com tireoidite auto-imune deve ser rigoroso pelo risco destes pacientes desenvolverem no curso de sua evolução um carcinoma papilar da tireóide ${ }^{22}$.

No futuro deveremos continuar avaliando a relação da tireoidite auto-imune e do carcinoma papilar da tireóide, porém, em uma população selecionada de pacientes com doença auto-imune sistêmica onde o risco de desenvolver neoplasias é maior que na população em geral $^{23}$ e onde a presença de tireoidite auto-imune pode chegar a $44,4 \%$ dos pacientes $^{24}$.

1. Existe uma relação de freqüência quatro vezes maior da tireoidite auto-imune com o carcinoma diferenciado da tireóide em comparação com outras patologias tireoidianas (16,7\% X 3,6\%).

2. Podemos inferir que a associação entre tireoidite auto-imune e carcinoma diferenciado da tireóide deva ser mais que casual (16,7\%), exigindo uma observação clínico-laboratorial cuidadosa dos portadores da doença autoimune.

\section{ABSTRACT}

Background: In spite of the controversial statistical relationship, the association of Hashimoto thyroiditis and differentiated carcinoma seems to be increasing. Objective: To assess the association of Hashimoto thyroidites and differentiated carcinoma at Santa Casa de Misericórdia de Goiânia, Goiás. Method: From 1395 thyroidectomies performed from 1994 to 2003,120 differentiated carcinoma (27 folicular and 93 papillary) were selected, analyzing clinical features (age and gender) with presentation of the frequency and a summary of measure resume in statistical description. For evaluation of auto-immune disease and differentiated carcinoma, contingence tables and analysis through non parametric test of Pearson $K$ Square testwere employedc, with a level of significance of $5 \%$ (p less than 0.05\%). Results: We found a frequency of association of four times between Hashimoto thyroiditis and differentiated carcinoma of the thyroid in relation to others thyroid pathologies (16.7\% versus 3.6\%). Conclusion: These results allow us the conclusion that this association in not casual, suggesting a clinical and laboratorial follow up in patients with auto-immune diseases.

Key words: Thyroiditis; Thyroid neoplasms; Thyroiditis, autoimmune. 


\section{REFERÊNCIAS}

1. Hedinger C. Geographic pathology of thyroid diseases. Pathol Res Pract. 1981;171(3-4):285-92.

2. Weaver DK, Batsakis JG, Nishiyama RN. Relationship of iodine to "lymphocytic goiters". Arch Surg. 1969;98(2):183-6.

3. Holm LE, Blomgren H, Lowhagen T. Cancer risks in patients with chronic lymphocytic thyroiditis. N Engl J Med. 1985;312(10):601-4.

4. Segal K, Ben-Bassat M, Avraham A, Har-El G, Sidi J. Hashimoto's thyroiditis and carcinoma of the thyroid gland. Int Surg. 1985;70(3):205-9.

5. Maceri DR, Sullivan MJ, McClatchney KD. Autoimmune thyroiditis: pathophysiology and relationship to thyroid cancer. Laryngoscope. 1986;96(1):82-6.

6. Singh B, Shaha AR, Trivedi H, Carew JF, Poluri A, Shah JP. Coexistent Hashimoto's thyroiditis with papillary thyroid carcinoma: impact on presentation, management and outcome. Surgery. 1999;126(10):1070-6; discussion 1076-7.

7. Mechler C, Bounacer A, Suarez H, Saint Frison M, Magois C, Aillet G, Gaulier A. Papillary thyroid carcinoma: 6 cases from 2 families with associated lymphocytic thyroiditis harbouring RET/ PTC rearrangements. Br J Cancer. 2001;85(12):1831-7.

8. Unger P, Ewart M, Wang BY, Gan L, Kohtz DS, Burstein DE. Expression of p63 papillary thyroid carcinoma and in Hashimoto's thyroiditis: a pathobiologic link? Hum Pathol. 2003;34(8):764-9.

9. Hirabayashi RN, Lindsay $\mathrm{S}$. The relation of thyroid carcinoma and chronic thyroiditis. Surg Gynecol Obstet. 1965;121:243-52.

10. Zois C, Stavrou I, Kalogera C, Svarna E, Dimoliatis I, Seferiadis $\mathrm{K}$, Tsatsoulis A. High prevalence of autoimmune thyroiditis in schoolchildren after elimination of iodine deficiency in northwestern Greece. Thyroid. 2003;13(5):485-9.

11. Feldt-Rasmussen U. Iodine and cancer. Thyroid. 2001;11(5):483-6.

12. Merhy J, Driscoll HK, Leidy JW, Chertow BS. Increasing incidence and characteristics of differentiated thyroid cancer in Huntington, West Virginia. Thyroid. 2001;11(11):1063-9.

13. Burgess JR. Temporal trends for thyroid carcinoma in Australia: an increasing incidence of papillary thyroid carcinoma (19821997). Thyroid. 2002;12(2):141-9.

14. Nagataki S, Nyström E. Epidemiology and primary prevention of thyroid cancer. Thyroid. 2002;12(10):889-96.
15. Delange F, De Benoist B, Pretell E, Dunn JT. Iodine deficiency in the world: where do we stand at the turn of the century? Thyroid. 2001;11(5):437-47.

16. Meier DW, Woolner LB, Beahrs OH, McConahey WM. Parenchymal findings in thyroidal carcinoma: pathologic study of 256 cases. J Clin Endocrinol Metab. 1959; 19(1):162-71.

17. Schlicke CP, Hill JE, Schultz GF. Carcinoma in chronic thyroiditis. Surg Gynecol Obstet. 1960;111:552-6.

18. Eisenberg BL, Hensley SD. Thyroid cancer with coexistent Hashimoto's thyroiditis. Clinical assessment and management. Arch Surg. 1989;124(9):1045-7.

19. Ott RA, MacCall AR, McHenry C, Jarosz H, Armin A, Lawrence AM, Paloyan E. The incidence of thyroid carcinoma in Hashimoto's thyroiditis. Am Surg. 1987;53(8):442-5.

20. Arif S, Blanes A, Diaz-Cano SJ. Hashimoto's thyroiditis shares features with early papillary thyroid carcinoma. Histopathology. 2002;41(4):357-62.

21. Little JW. Thyroid disorders. Part II: hypothyroidism and thyroiditis. Oral Surg Oral Med Oral Pathol Oral Radiol Endod. 2006;102(2):148-53. Epub 2006 Jun 19.

22. Little JW. Thyroid disorders. Part III: neoplastic thyroid disease. Oral Surg Oral Med Oral Pathol Oral Radiol Endod. 2006;102(3):275-80.

23. Cibere J, Sibley J, Haga M. Systemic lupus erythematosus and the risk of malignancy. Lupus. 2001;10(6):394-400.

24. Mihailova D, Grigorova R, Vassileva B, Mladenova G, Ivanova N, Stephanov S, Lissitchky K, Dimova E. Autoimmune thyroid disorders in juvenile chronic arthritis and systemic lupus erythematosus. Adv Exp Med Biol. 1999;455:55-60.

Como citar este artigo:

Roberti A, Sobrinho JA, Denardin OVP, Rapoport A. Concomitância da tireoidite de Hashimoto e o carcinoma diferenciado da tireóide. Rev Col Bras Cir. [periódico na Internet] 2006 Set-Out;33(5). Disponível em URL: www.scielo.br/rcbc

Endereço para correspondência:

Prof. Dr. Abrão Rapoport

Rua Iramaia, $n^{\circ} 136-\mathrm{Jd}$. Europa

01450-020 - São Paulo - Brasil

E-mail: arapoport@terra.com.br 